\title{
Hochschule
}

Gerhard Reichmann*, Christian Schlögl und Stefan Thalmann

\section{Das Institut für Informationswissenschaft an der Universität Graz: 1987 - 2020}

https://doi.org/10.1515/iwp-2020-2132

Zusammenfassung: Die Geschichte des Instituts für Informationswissenschaft an der Universität Graz in Österreich von seiner Gründung 1987 bis zur Fusionierung mit anderen Instituten im Jahr 2020 wird beschrieben und mit Daten aus bibliometrischen Analysen der Publikationen ergänzt. Abschließend wird ein Ausblick auf die Einbindung der Informationswissenschaft in neue Studienangebote gegeben.

Deskriptoren: Universität Graz, Institut für Informationswissenschaft, Österreich, Geschichte, Lehre, Forschung, Bibliometrie

The Institute for Information Science at the University of Graz: 1987 - 2020

Abstract: The history of the Institute for Information Science at the University of Graz in Austria from its foundation in 1987 until its merger with other institutes in 2020 is described and supplemented with data from bibliometric analyses of publications. Finally, the integration of information science in new study programmes is described.

Descriptors: University of Graz, Institute for Information Science, Austria, history, teaching, research, bibliometrics

*Kontaktperson: Ao. Prof. DDr. Gerhard Reichmann, Karl-Franzens-Universität Graz, Institut für Operations und Information Systems, Universitätsstraße 15, F3, 8010 Graz, E-Mail: gerhard.reichmann@uni-graz.at Ao. Prof. Dr. Christian Schlögl, Karl-Franzens-Universität Graz, Institut für Operations und Information Systems, Universitätsstraße 15/F3, 8010 Graz, E-Mail: christian.schloegl@uni-graz.at Prof. Dr. Stefan Thalmann, Karl-Franzens-Universität Graz, Institut für Operations und Information Systems, Universitätsstraße 15/F3, 8010 Graz, E-Mail: stefan.thalmann@uni-graz.at

\author{
L'Institut des sciences de l'information de l'Université de \\ Graz : 1987 - 2020
}

Résumé: L'histoire de l'Institut des sciences de l'information de l'Université de Graz en Autriche, depuis sa fondation en 1987 jusqu'à sa fusion avec d'autres instituts en 2020, est décrite et complétée par des données issues d'analyses bibliométriques des publications. Enfin, une perspective sur l'intégration des sciences de l'information dans les nouveaux cours d'études est donnée.

Descripteurs: Université de Graz, Institut des sciences de l'information, Autriche, histoire, enseignement, recherche, bibliométrie

\section{Historischer Überblick}

Im Jahr 1987 wurde das Institut für Informationswissenschaft an der Karl-Franzens-Universität Graz gegründet. $\mathrm{Zu}$ Beginn umfasste die personelle Grundausstattung neben der Professorenstelle (Lehrstuhlinhaber: Wolf Rauch) drei wissenschaftliche Mitarbeitende und eine Schreibkraft. In den Jahren 1990 bzw. 1997 wurden zwei dieser drei wissenschaftlichen Stellen mit Personen besetzt, deren Dienstverhältnisse nach einigen Jahren (nach erfolgter Dissertation und Habilitation) verstetigt wurden, und die damit ebenfalls zum „Stammpersonal“ des Instituts zählen. Eine Besonderheit des Instituts bestand darin, dass es an einer Sozial- und Wirtschaftswissenschaftlichen Fakultät eingerichtet wurde. ${ }^{1}$ Es bot daher kein eigenes informationswissenschaftliches Studium an, die Lehrveranstaltungen konnten vielmehr von allen Studierenden der Sozial- und Wirtschaftswissenschaften im Rahmen ihrer Spezialisierung (dies galt insbesondere für Studierende der Betriebswirtschaftslehre und der Wirtschaftspädagogik) oder als Wahl(pflicht)fächer belegt werden. Dies hatte auch Einfluss auf die Institutsausrichtung, die von Anfang an einen

1 In Deutschland sind bzw. waren informationswissenschaftliche Institute meist in einem geisteswissenschaftlichen Umfeld angesiedelt, wie etwa an den Universitäten Düsseldorf und Hildesheim. 
starken wirtschaftswissenschaftlichen Bezug hatte. Neben den Bereichen „Information und Dokumentation“ umfasste diese in Forschung und Lehre auch die Themenfelder „Informationsmanagement“ und „Wirtschaftsinformatik“.

Nach einem Ruf an die Humboldt-Universität zu Berlin konnte der Lehrstuhlinhaber im Zuge der Abwehrverhandlungen für das Institut ab 1996 noch eine weitere wissenschaftliche Mitarbeiterstelle sowie einen IT-Techniker gewinnen. Im Jahr 2007 erfolgte eine Umbenennung des Instituts in Institut für Informationswissenschaft und Wirtschaftsinformatik. Dadurch wurde dem Umstand Rechnung getragen, dass es an der Sozial- und Wirtschaftswissenschaftlichen Fakultät kein Wirtschaftsinformatik-Institut gab. Forschung und Lehre sollten in diesem Bereich verstärkt werden. Dazu wurde eine weitere Mitarbeiterstelle verstetigt und fakultätsintern mit einem habilitierten Wirtschaftsinformatiker besetzt. So umfasste ab diesem Zeitpunkt das wissenschaftliche „Stammpersonal“ des Instituts den Lehrstuhlinhaber sowie drei weitere habilitierte Personen.

Als einziges facheinschlägiges Universitätsinstitut in Österreich hatte das Institut eine zentrale Rolle im Bereich der Informationswissenschaft inne. Beispielsweise wirkte einer der zum „Stammpersonal“ zählenden wissenschaftlichen Mitarbeiter am Aufbau des damaligen Fachhochschul-Studiengangs für Informationsberufe in Eisenstadt mit. Auch bei internationalen Forschungsprojekten und anderen internationalen Aktivitäten war das Institut erste Ansprechstelle in Österreich.

Mit dem 1. Oktober 2020 endete die Geschichte des Instituts für Informationswissenschaft als selbständige Organisationseinheit, indem dieses gemeinsam mit zwei weiteren Instituten der Sozial- und Wirtschaftswissenschaftlichen Fakultät, dem Institut für Statistik und Operations Research sowie dem Institut für Produktion und Logistik, in das neue Institut für Operations und Information Systems überführt wurde. Grund für diese Fusionierung waren strategische Überlegungen der Fakultät, die bestrebt ist, größere Organisationseinheiten zu bilden, um Synergieeffekte zu generieren. Die Auswahl der drei zusammengelegten Institute erfolgte nach inhaltlichen, personellen und räumlichen Aspekten: Zwei der drei fusionierten Institute, nämlich das Institut für Informationswissenschaft und das Institut für Statistik und Operations Research, gehörten nicht zu den betriebswirtschaftlichen „Kerninstituten“. Daneben erfolgte die Zusammenlegung zum Zeitpunkt der Emeritierung des Leiters des Instituts für Informationswissenschaft; dessen Nachfolger ist ein Wirtschaftsinformatiker. An allen drei Instituten gibt es einen relativ hohen Anteil an dauerhaft angestellten Personen, die in nicht allzu ferner Zukunft in Pension gehen werden, sodass die lang- fristige Ausrichtung des neuen Instituts durchaus flexibel ist. Und schließlich befanden sich alle drei Institute in unmittelbarer Nachbarschaft zueinander, sodass keine räumlichen Veränderungen notwendig waren.

Nachfolgend wird zunächst auf Forschung und Lehre des Instituts während seines Bestehens eingegangen. Danach erfolgt ein Ausblick auf das „Nachfolgeinstitut“. Schließlich wird beschrieben, wo informationswissenschaftliche Inhalte in Zukunft an der Universität Graz weiterhin $\mathrm{zu}$ finden sind.

\section{Forschung}

In diesem Abschnitt wird die Forschungstätigkeit des Instituts dargelegt. Zunächst wird ein Überblick über die wichtigsten Forschungsfelder und anschließend auf die Publikationstätigkeit gegeben, wobei sich die Publikationsanalyse auf die Forschungstätigkeit des „Stammpersonals“, also der vier habilitierten Mitarbeiter mit unbefristeten Dienstverhältnissen, ab dem jeweiligen Beschäftigungsbeginn am Institut beschränkt. Die zeitlich befristet Beschäftigten nutzten die vier Jahre als Angestellte vorwiegend dazu ihre Dissertationen zu verfassen, bei denen es sich sehr häufig um nicht publizierte Monografien handelte. Somit hat es von dieser Seite nur relativ wenige Publikationsaktivitäten gegeben.

Tabelle 1 zeigt, dass sich die Forschung am Institut für Informationswissenschaft (für einen Überblick bzgl. der Forschungsaktivitäten: vgl. Dorsch et al. 2017, Schlögl 2013 und Bauer et al. 2005) bei weitem nicht auf den Bereich der Informationswissenschaft beschränkte, sondern neben der Wirtschaftsinformatik auch noch weitere Forschungsfelder umfasste, die sich u. a. aufgrund von Praxis- und/ oder Kooperationsprojekten mit Forschenden aus anderen Bereichen ergaben. Zahlreiche Publikationen belegen diese Aktivitäten in den Forschungsfeldern „Evaluierung“ (vgl. z.B. Reichmann 2008) und „Gesundheitsökonomie“ (vgl. z. B. Reichmann \& Sommersguter-Reichmann 2012).

Zentrale Forschungsfelder im Kernbereich der Informationswissenschaft waren „Bibliotheksmanagement“ (vgl. z. B. Reichmann 2004 und Rauch 2000), „Informetrie und Szientometrie“ (vgl. z.B. Schlögl 2014), „Informationsdynamik“ (vgl. z. B. Rauch 2016), „Informationskompetenz“ (vgl. z.B. Maurer, Schlögl \& Dreisiebner 2016), „Informationsrecht“ (vgl. z. B. Reichmann 2013), „Informations- und Wissensmanagement" (vgl. z.B. Schlögl 2003) und „Interkultureller Vergleich des Nutzungsverhaltens digitaler Medien“ (vgl. z. B. Luidold, Seo \& Schlögl 2016). Insgesamt betreffen mehr als 50 Prozent der Publikationen des Instituts diesen Kernbereich. 
Tabelle 1: Forschungsbereiche und Forschungsfelder.

\begin{tabular}{ll}
\hline Forschungsbereich & Forschungsfeld \\
\hline & Bibliotheksmanagement (Leistungsmes- \\
sung und Benutzerforschung) & Informetrie und Szientometrie \\
& Informationsdynamik \\
& Informationskompetenz \\
Informationswissenschaft & Informationsrecht (Datenschutz- und \\
& Urheberrecht) \\
& Informations- und Wissensmanagement \\
& Interkultureller Vergleich des \\
& Nutzungsverhaltens digitaler Medien \\
\hline \multirow{3}{*}{ Wirtschaftsinformatik } & Akzeptanz von Informations- und \\
& Kommunikationssystemen \\
& Geschäftsmodellinnovationen durch \\
& Digitale Kommunikationstechnologien \\
& Kommunikationsverhalten in der Welt \\
& des Digitalen \\
\hline \multirow{2}{*}{ Sonstiges } & Evaluierung (Non-Profit-Bereich) \\
& Gesundheitsökonomie \\
\hline
\end{tabular}

Die Wirtschaftsinformatik hat nicht erst seit der Umbenennung des Instituts im Jahre 2007, sondern schon von jeher eine zentrale Rolle in der Forschung des Instituts gespielt (vgl. z.B. Schlögl 2004). Bearbeitete Forschungsfelder waren „Akzeptanz von Informations- und Kommunikationssystemen“ (vgl. z. B. Petrovic \& Platzer 2009), „Geschäftsmodellinnovationen durch Digitale Kommunikationstechnologien“ (vgl. z. B. Petrovic, Puchleitner \& Harnisch 2014) sowie „Kommunikationsverhalten in der Welt des Digitalen“ (vgl. z. B. Platzer, Petrovic \& Rauch 2010).

Eine Publikationsanalyse für die Jahre 1987 bis 2019 legt offen, dass in diesem Zeitraum vom „Stammpersonal“ des Instituts bei Vollzählung (Normal Counting-Verfahren) insgesamt 333 Publikationen verfasst wurden (Herausgeberschaften und graue Literatur wurden nicht berücksichtigt). ${ }^{2}$ Dies ergibt eine durchschnittliche Anzahl von ca. zehn Publikationen pro Jahr. Aufschluss über die zeitliche Verteilung dieser Publikationen gibt Abbildung 2 (blaue Linie): Demnach hat es erhebliche jahresweise Schwankungen gegeben, der Trend ist aber deutlich steigend (vgl. dazu die blau gepunktete Trendlinie in Abb. 2). Dies relativiert sich dadurch, dass die Anzahl des „Stammpersonals“ des Instituts, von ursprünglich einer Person, dem Lehrstuhlinhaber, bis 2007 auf vier Personen gewachsen ist (1990 auf zwei und 1997 auf drei Personen); d.h. nur die Jahre 2007 bis 2019 sind unter Berücksichti-

2 Das Jahr 2020, mit bisher nur einer Publikation, ist in den Abbildungen enthalten, wird aber außer Acht gelassen, weil hierfür zum Zeitpunkt der Datenerhebung noch keine vergleichbaren Daten vorlagen; zudem existierte das Institut nur bis September 2020. gung der vorhandenen personellen Ressourcen unmittelbar miteinander vergleichbar. Die durchschnittliche Anzahl an Publikationen pro Jahr beträgt für diesen Zeitraum 16. Das publikationsstärkste Jahr war eindeutig 2013 mit 34 Publikationen, gefolgt von den Jahren davor und danach mit je 24 Publikationen. Danach hat der Publikationsoutput erkennbar abgenommen, weil zunehmend versucht wurde, in möglichst renommierten Publikationsmedien zu veröffentlichen; mit der Folge, dass ein immer längerer Zeitraum zwischen Fertigstellung und Veröffentlichung eines Beitrages liegt. So warten derzeit ca. zehn fertig gestellte Beiträge auf ihre Veröffentlichung.

Wesentlich aussagekräftiger als die Gesamtzahl der Publikationen sind vermutlich die Zahlen auf Basis der Aufgliederung nach Publikationsarten. So lassen sich die insgesamt 333 Publikationen in drei Monografien, 130 Beiträge in wissenschaftlichen Zeitschriften, 115 Beiträge in Sammelwerken sowie 85 sonstige Beiträge (bsp. Buchbesprechungen, Editorials, Würdigungen, Lexikonbeiträge oder auch publizierte Abstracts) untergliedern. Die zeitliche Verteilung der Veröffentlichungen je Publikationsart ist in Abbildung 1 dargestellt. Auch hier zeigen sich deutliche Schwankungen. Besonders hoch sind diese bei den sonstigen Beiträgen, was v.a. an den zahlreichen Lexikonbeiträgen in den Jahren 2013 und 2014 liegt. Der Schwerpunkt der Publikationstätigkeit des Instituts lag eindeutig auf Beiträgen in Zeitschriften und in Sammelwerken, insbesondere Kongressbänden, mit durchschnittlich 3,9 bzw. 3,5 Beiträgen pro Jahr. Zahlenmäßig spielen die drei im Betrachtungszeitraum veröffentlichten Monografien kaum eine Rolle, bei Betrachtung der Anzahl der veröffentlichten Seiten dagegen sehr wohl: 665 der insgesamt 3.540 publizierten Seiten entfielen auf diese Publikationsart, weitere 1.249 bzw. 1.226 auf Beiträge in Zeitschriften bzw. Sammelwerken und nur 400 auf sonstige Beiträge.

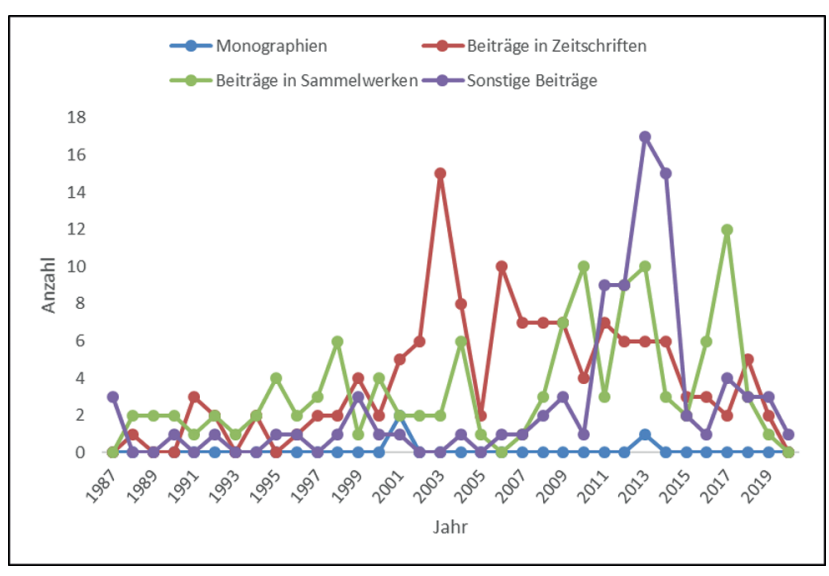

Abbildung 1: Publikationsarten. 
Während die bisher vorgenommenen Analysen zur Publikationstätigkeit auf dem Normal Counting-Verfahren beruhen, das bei Mehrfachautorenschaften jede Publikation für jeden beteiligten Autor „voll“ zählt, also mit eins bewertet, kommt bei institutionalisierten Forschungsevaluierungen bevorzugt das Fractional Counting-Verfahren zum Einsatz, bei dem die Publikationen den beteiligten Autoren nur anteilsmäßig, bevorzugt gleichverteilt nach der Formel 1/n, zugerechnet werden. Nachdem am Institut für Informationswissenschaft viele Publikationen in Alleinautorenschaft verfasst wurden, führt die Heranziehung des zweitgenannten Verfahrens zu keinen gravierenden Veränderungen (s. Abb. 2). Dennoch würde der Forschungsoutput im Betrachtungszeitraum von $333 \mathrm{Pu}-$ blikationen auf 269 „Publikationsanteile“ sinken. Anhand von Abbildung 3 lässt sich erkennen, dass der Anteil der Publikationen in Koautorenschaft (d.h. für alle Publikationsarten) relativ starken jährlichen Schwankungen unterworfen ist. Im Durchschnitt liegt dieser bei 32 Prozent, d.h. ca. ein Drittel aller Publikationen wurde gemeinsam mit anderen verfasst. Betrachtet man die einzelnen Publikationsarten, so hatten Beiträge in Zeitschriften mit durchschnittlich 43 Prozent den höchsten Anteil an Koautorenschaften. Die entsprechenden Werte für Monografien, Beiträge in Sammelwerken und sonstige Beiträge betragen 33 Prozent, 37 Prozent und neun Prozent.



Abbildung 2: Publikationen: Normal Counting versus Fractional Counting $(1 / n)$.

Der Anteil an englischsprachigen Publikationen (s. Abb. 3, vgl. rot gepunktete Trendlinie) ist stark steigend und liegt bei 31 Prozent, wobei bis 2002 insgesamt zehn Jahre keine und zwischen 2015 und 2019 mehr als 80 Prozent der Publikationen englischsprachig waren. Hier ist der Anteil bei Beiträgen in Sammelwerken mit 45 Prozent am höchsten, gefolgt von Zeitschriftenartikeln (26\%) und sonstigen Beiträgen (16\%). Alle drei Monografien wurden in deutscher Sprache verfasst.

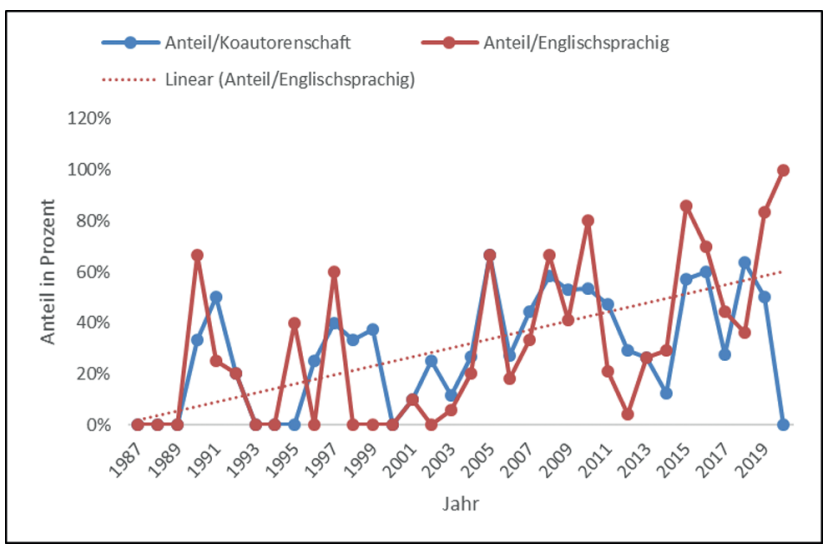

Abbildung 3: Anteil der Publikationen in Koautorenschaft bzw. in englischer Sprache.

Beiträge in wissenschaftlichen Zeitschriften sind - aus Sicht der Autoren - mit Abstand die wichtigste Publikationsart (s. Abb. 4). Diese wurden in den Anfangsjahren des Instituts vorwiegend in Alleinautorenschaft und auf Deutsch verfasst.

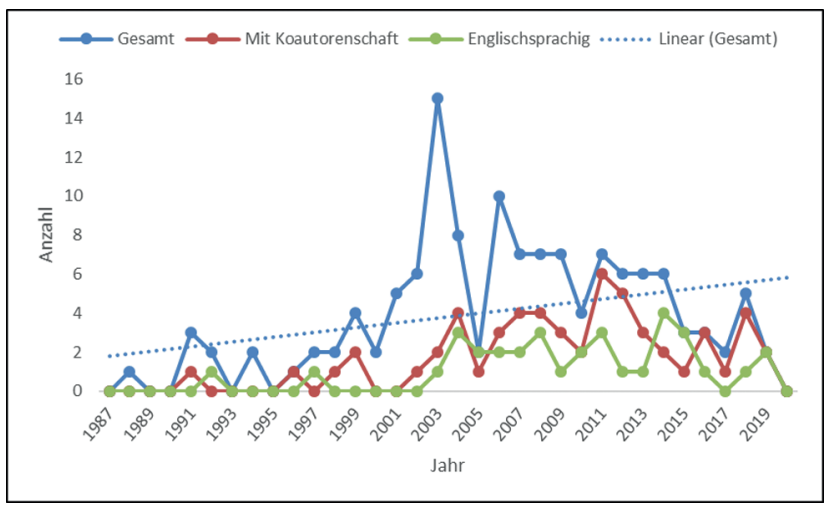

Abbildung 4: Beiträge in Zeitschriften.

Die zahlreichen Texte in Konferenzbänden belegen, dass Mitarbeitende des Instituts auch häufig an Konferenzen teilgenommen und Vorträge gehalten haben. Das Institut wirkte zudem an der Organisation bzw. Durchführung von wissenschaftlichen Tagungen aktiv mit. Im Jahr 1994 hat es das 4. Internationale Symposium für Informationswissenschaft in Graz (ISI 1994) organisiert. Beim 14. Internationalen Symposium für Informationswissenschaft (ISI 2015) war ein Institutsmitglied Leiter des Programmkomitees. Und schließlich war ein Mitarbeiter des Instituts Mitglied im Programmkomitee des ASIST Annual Meeting (AM 2016) in Kopenhagen.

Auch in der internationalen Zeitschriftenkommunikation spielt das Institut eine gewisse Rolle. So ist ein Institutsmitglied im Herausgeberbeirat von IWP-Information, Wissenschaft und Praxis, Liber Quarterly und Journal of 
Information Science Theory and Practice, die übrigen Institutsmitglieder sind regelmäßig als Gutachter für einschlägige Fachzeitschriften tätig.

\section{Lehre}

Dieser Abschnitt ist der Lehrtätigkeit des Instituts gewidmet und umfasst zunächst einen kurzen Einblick in Umfang und Ausrichtung der Lehre auf Basis der relevanten Studienpläne. Anschließend wird auf die Intensität der Inanspruchnahme der angebotenen Lehre seitens der Studierenden eingegangen.

Wie bereits erwähnt, war das Institut für Informationswissenschaft an einer Sozial- und Wirtschaftswissenschaftlichen Fakultät angesiedelt. Die Lehre des Instituts war stets auf die an dieser Fakultät angebotenen Studiengänge ausgerichtet, wobei die Betriebswirtschaftslehre (BWL) aufgrund der starken Einbeziehung der informationswissenschaftlichen (Lehr)Ressourcen sowie der großen Anzahl an Studierenden von zentraler Bedeutung war. Das Lehrangebot des Instituts umfasste immer mindestens eine „Spezialisierung“ im Rahmen des jeweiligen betriebswirtschaftlichen Studiums an dieser Fakultät (vgl. dazu auch Reichmann 2012). Dies ist insofern bemerkenswert, als die Studierenden oftmals an dieser Stelle eine entscheidende Weichenstellung für die spätere Berufstätigkeit vornehmen. Zudem wurden und werden die Themen der wissenschaftlichen Abschlussarbeiten (früher Diplom-, heute Masterarbeiten) üblicherweise diesen Spezialisierung(en) entnommen. Somit konkurrierte das Angebot des Instituts für Informationswissenschaft mit denen „klassischer“ betriebswirtschaftlicher Institute, wie der Institute für Rechnungswesen (Accounting), Finanzwirtschaft oder auch Marketing, was eine große Herausforderung war. Umso erfreulicher, dass das Spezialisierungsangebot des Instituts stets auf Nachfrage durch Studierende stieß (vgl. dazu auch Reichmann 2007). Stundenmäßig umfasste es, je nach gerade gültigem Studienplan, zwischen 14 und 20 Semesterwochenstunden, die fast durchgängig - jedes Semester angeboten wurden. Inhaltlich gab es eine Weiterentwicklung von traditionellen informationswissenschaftlichen Themen, wie etwa „Informationsmanagement“, „Dokumentation“ oder „Information Retrieval“, zu aktuellen informationswissenschaftlichen und zunehmend auch wirtschaftsinformatischen Lehr- und Forschungsfeldern, wie etwa „Informationskompetenz“, „Soziale Medien“, „Business Intelligence“, „Digitale Ökonomie“ oder auch „Business Analytics“.

Neben den Lehrveranstaltungen im Rahmen der Spezialisierung wurden jedes Semester zahlreiche weitere
Lehrveranstaltungen angeboten, die von den Studierenden als Wahlfächer besucht werden konnten. Das Angebot umfasste z.B. im letzten Jahr des Bestehens des Instituts u. a. Lehrveranstaltungen zu den Themen „SAP“, „SPSS“, „Informationsrecht“ oder auch „Projektmanagement“.

Dieses umfangreiche Lehrangebot konnte nicht vom „Stammpersonal“, dessen Lehrdeputat im Durchschnitt ca. sieben Semesterwochenstunden umfasste, sowie den befristet beschäftigten Institutsmitarbeitenden mit rund vier Semesterwochenstunden Lehre abgedeckt werden. Deshalb wurde stets auf externe Lehrende, großenteils aus der Praxis, zurückgegriffen.

$\mathrm{Zu}$ den Studierendenzahlen existieren nur wenige exakte historische Daten, so etwa für die ersten drei Jahre des Instituts, in denen 66 (Studienjahr 1987/88), 95 (Studienjahr 1988/89) bzw. 128 (Studienjahr 1989/90) Personen mit Informationswissenschaft als Spezialisierung begannen. Allerdings gab es damals viele Studienabbrecher, sodass diese Zahlen kaum Rückschlüsse zulassen, wie viele Studierende die gewählte Spezialisierung abgeschlossen haben. Generell waren bis ca. zum Jahr 2010 die Lehrveranstaltungen des Instituts mit begrenzter Teilnehmerzahl (in der Regel 25 bis 30 Studierende) mehr oder weniger voll belegt. Seit damals sind die Studierendenzahlen an der Sozial- und Wirtschaftswissenschaftlichen Fakultät der Universität Graz infolge des wachsenden Studienangebots an den Fachhochschulen sowie des zunehmenden Eintritts von geburtenschwächeren Jahrgängen ins Studienalter stark zurückgegangen. Dies wirkte sich auch auf die Teilnahmerzahlen an den Lehrveranstaltungen aus, die sich bis 2020 praktisch halbierten. Um diesem Trend entgegenzusteuern, wurden die Lehrveranstaltungen der Spezialisierung im Bachelorstudium seit einigen Jahren in englischer Sprache angeboten, um nicht-deutschsprachige Austauschstudierende (v.a. solche im Erasmus-Programm) zu gewinnen. Diese Strategie war großteils von Erfolg gekrönt.

Ein wichtiger Bestandteil der Lehre am Institut für Informationswissenschaft war die Betreuung der wissenschaftlichen Arbeiten. Insgesamt wurden vom „Stammpersonal“ des Instituts im Betrachtungszeitraum 399 Diplom- bzw. Masterarbeiten sowie 69 Dissertationen betreut, ein Jahresschnitt von ca. zwölf Diplom- bzw. Masterarbeiten und zwei Dissertationen. Dafür stand bis zum Jahr 2000 nur der Lehrstuhlinhaber als einzige habilitierte Person zur Verfügung. Deshalb ist es nicht überraschend, dass es bei den jährlich betreuten Diplom- bzw. Masterarbeiten einen deutlichen Anstieg gab (Abb. 5). In drei Jahren (2010, 2015 und 2016) wurden sogar mehr als 20 Arbeiten betreut. Die Dissertationen unterlagen deutlichen Schwankungen, wobei sich die hohen Zahlen in den 
Jahren 2003 und 2011 v.a. aus den vielen (wesentlich weniger aufwendigen) Zweitbetreuungen ergaben. Insgesamt halten sich Erst- und Zweitbetreuungen ziemlich genau die Waage.

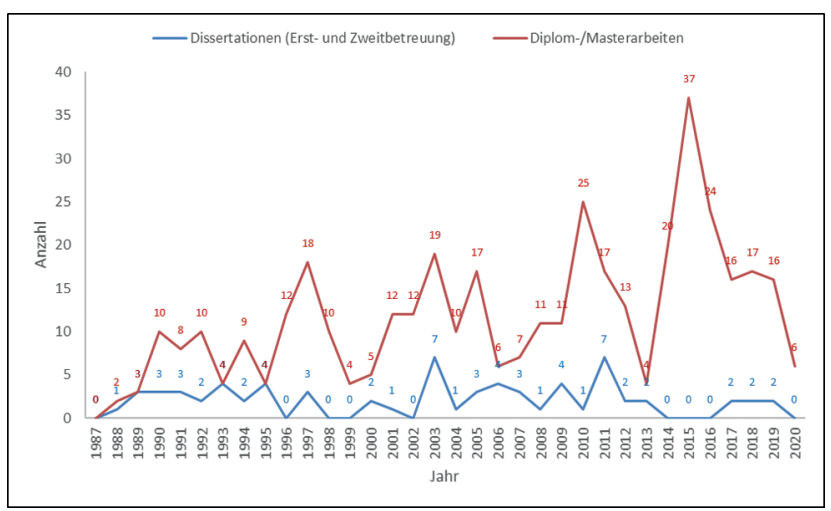

Abbildung 5: Betreute Diplom-/Masterarbeiten und Dissertationen.

Seit dem Studienjahr 2014/15 bot das Institut für Informationswissenschaft gemeinsam mit dem Institut für Informationswissenschaft der Universität Hildesheim und zwei Koreanischen Partneruniversitäten (Pai Chai University und Chungbuk National University) das Joint Degree Masterstudium Global Studies on Management and Information Science an (GLOMIS o.D.), das Studierenden der europäischen/koreanischen Partneruniversitäten ermöglichte, ein Jahr in Korea bzw. Österreich oder Deutschland zu studieren. Dieses Angebot ist allerdings befristet und so haben im Studienjahr 2020/21 Studierende das letzte Mal die Möglichkeit, sich für GLOMIS einzuschreiben.

\section{Ausblick}

Ein Blick in die Zukunft lässt sich mit folgendem Satz gut charakterisieren: die Informationswissenschaft ist tot, lang lebe die Informationswissenschaft! Durch die Fusionierung gibt es in Österreich kein Institut mehr, das die Bezeichnung „Informationswissenschaft“ im Titel trägt. Dies bedeutet aber nicht, dass informationswissenschaftliche Themen in Zukunft nicht mehr behandelt werden. An der Universität Graz sollte dies durch folgende drei „Einrichtungen“ sichergestellt werden:

- Arbeitsgruppe „Information Systems“ und BANDASCenter

- Forschungsnetzwerk „Human Factor in Digital Transition" (HFDT)

- Inter-universitärer postgradualer Universitätslehrgang „Library and Information Studies“

\subsection{Arbeitsgruppe „Information Systems“ und BANDAS-Center}

Wenn man die Entwicklung von Google und Facebook \& Co. betrachtet, dann ist es naheliegend, eine informationswissenschaftliche Professur mit einem „Data Scientist“ nachzubesetzen. Wird die Informationswissenschaft traditionell als interdisziplinäre Perspektive (Informatik, Bibliothekswissenschaften, Linguistik, Psychologie) auf den Prozess der Informationssammlung, -speicherung, -organisation, -suche, -interpretation und -nutzung gesehen, so betrachtet Data Science diesen Prozess deutlich stärker aus der technischen Automatisierungsperspektive und mit dem Fokus auf Big Data (Provost \& Fawcett 2013). Da die Informationswissenschaft sehr heterogen und interdisziplinär ausgerichtet ist, lassen sich sehr viele verschiedene Anwendungsfelder, darunter auch Data Science und Information Systems, finden (Kolp, Snoeck, Vanderdonckt \& Wautelet 2019; Dalpiaz 2020). In diesen wird das Potential der modernen Data-Science-Methoden auf klassische informationswissenschaftliche Fragestellungen betrachtet und ein klarer Bezug hergestellt (Virkus \& Garoufallou 2019). Gelegentlich wird Data Science sogar als Teilthemengebiet der Informationswissenschaft interpretiert (Marchionini 2017). Forschende sehen Informationswissenschaft und Data Science als eng verwobene Disziplinen mit großen Überlappungsfeldern (Wang 2018).

Für die Informationswissenschaft stellt sich die Frage, welche Auswirkung der stärkere Bedeutungsgewinn von Data Science haben wird. Sind ähnlich disruptive Folgen zu erwarten, wie der Wandel von den praktischen Bibliotheksthemen zu wissenschaftlichen Themen rund um Information und ihrer Nutzung in den 1960ern, oder der stärker interdisziplinären Ausrichtung an Wirtschafts- und Informatikthemen der 1990er (Larivière, Sugimoto \& Cronin 2012; Dora \& Kumar 2019)? Fragestellungen der Data Science und Information Systems sind in der Informationswissenschaft nicht neu, sondern treten vor allem auf Grund aktueller technischer und gesellschaftlicher Entwicklungen stärker in den Vordergrund. So stellen Hsiao und Chen (2020) eine zunehmende Überschneidung der Themenfelder aus den Bereichen „Data Science“ und „Informationswissenschaft" fest. Diese Annäherung findet sich nicht nur in der Forschung, sondern auch in Curricula und Studiengängen (Ortiz-Repiso, Greenberg \& Calzada-Prado 2018). So ist anzumerken, dass Expertise und Zugang aus Informationswissenschaft und Data Science nicht widersprüchlich sind, sondern sich ganz im Gegenteil sowohl in der Forschung als auch in der Lehre sehr gut ergänzen.

An der Fakultät wurde bereits vor einem Jahr das Business Analytics und Data Science (BANDAS) Center ge- 
gründet. Dieses universitäre Forschungszentrum soll fakultätsintern als Kompetenzzentrum fungieren und Kontakte zu Wirtschaft und Gesellschaft intensivieren. Die erste Aufgabe des BANDAS-Centers ist, den Einsatz und die Anwendung datenbasierter Technologien in den BWL-Anwendungsfeldern $\mathrm{zu}$ untersuchen. Es hat damit eine natürliche Schnittstelle zu allen BWL-Fachbereichen. Die zweite wichtige Aufgabe des BANDAS-Centers ist, die gesellschaftlichen Auswirkungen datenbasierter Technologien zu erforschen und somit die sozialwissenschaftliche Perspektive der Fakultät zu vertreten. Das BANDAS-Center wird sowohl räumlich als auch personell eng mit dem neuen Institut und speziell mit der Arbeitsgruppe „Information Systems" verbunden sein.

Mit dem stärkeren Anwendungsbezug der Arbeitsgruppe ist auch eine stärkere Ausrichtung auf Informatikthemen verbunden. Da die Universität Graz keine eigene Informatik-Arbeitsgruppe hat, wurde dazu eine enge Kooperation mit der Fakultät für Informatik der Technischen Universität Graz aufgebaut. Aus dieser Kooperation zwischen den Fakultäten ist auch ein neuer Masterstudiengang „Computational Social Systems“ mit den Majors „Business Analytics“ (BWL-Informatik), „Law and Computer Science“ (Rechtswissenschaften - Informatik), „Human Factors“ (Psychologie - Informatik) und „Social Research and Technologies“ (Soziologie - Informatik) hervorgegangen. Hier wird das Institut und speziell die Arbeitsgruppe „Information Systems“ eine wichtige koordinierende Rolle für dieses Studium und den Major „Business Analytics“ einnehmen. Dieser Fokus wird auch zusammen mit dem Institut für Entrepreneurship für die Managementausbildung aufgegriffen und ein MBA-Programm „Digital Leadership“ entwickelt. Durch das deutlich größere Lehrportfolio gibt es nach wie vor reichlich Platz für informationswissenschaftliche Themen, die durch ihre traditionell interdisziplinäre Ausrichtung gut in das Spannungsfeld zwischen Technologie, wirtschaftswissenschaftlichen Anwendungen und Gesellschaft passen.

Forschungsseitig werden die bestehenden informationswissenschaftlichen Themen durch mehr technik-orientierte Wirtschaftsinformatikthemen ergänzt. Dies passiert durch die Anbindung des BANDAS-Centers an das Institut und durch die stärkeren Kooperationen mit der TU Graz. Ebenso sollen durch die Kooperationen mit den anderen beiden Arbeitsgruppen des Institutes (Operations Research und Supply-Chain Management), die ebenso eine starke technische Ausrichtung haben, weitere Impulse in diese Richtung erfolgen. Da auch in der Informationswissenschaft technische Themen immer stärker Einzug halten, wird dieser Trend auch die informationswissenschaftlichen Themen befruchten.

\subsection{Forschungsnetzwerk „Human Factor in Digital Transition" (HFDT)}

An der Universität Graz ist HFDT seit Anfang 2019 als eines von vier sogenannten Forschungsnetzwerken eingerichtet. Ziel von HFDT ist es zu untersuchen, ,... wie die Digitalisierung den Menschen und sein Handeln sowie sein Leben beeinflusst“" (Uni Graz, o.D.). Auch wenn der Fokus auf den Auswirkungen der Digitalisierung auf den Menschen liegt, so ist eine Reihe von (möglichen) Forschungsthemen auch informationswissenschaftlicher Natur. Dies kommt durch die am Netzwerk beteiligten Institutionen bzw. Forschungsbereiche zum Ausdruck:

- Operations and Information Systems: Data Science and Business Analytics

- Volkswirtschaftslehre: Informationsökonomie

- Soziologie: Informationsgesellschaft

- Rechtswissenschaften: Informationsrecht

- Geisteswissenschaften: digitale Geisteswissenschaften

- Theologie und Philosophie: Informationsethik

- Psychologie

- Pädagogik: digitales Unterrichtsdesign.

Die interdisziplinäre Zusammenarbeit innerhalb des Forschungsnetzwerkes erfolgt in folgenden zentralen Bereichen (HFDT, o.D.):

- Forschungsprojekte: Teilnahme an internationalen Projektausschreibungen und Durchführung von interdisziplinären Forschungsprojekten

- Regelmäßiges Brown-Bag Seminar

- Lehre: gemeinsames Doktorandenkolloquium

- Veranstaltungen: Abhaltung von Workshops und Symposien.

\subsection{Inter-universitärer postgradualer Universitätslehrgang „Library and Information Studies"}

Im Bereich der Lehre werden informationswissenschaftliche Inhalte zusätzlich durch den Universitätslehrgang „Library and Information Studies“3 ${ }^{3}$ abgedeckt. Es handelt sich dabei um einen inter-universitären Universitätslehrgang, der aktuell an den Universitäten Graz, Innsbruck und Wien sowie an der Österreichischen Nationalbiblio-

3 Ein umfassender Überblick über die Ausbildung von wissenschaftlichen Bibliothekaren in Österreich findet sich in Hörzer et al. (2020). 
thek in Kooperation mit der Universität Wien eingerichtet ist. Der erste Teil (Grundlehrgang) muss vom wissenschaftlichen Bibliothekspersonal an Universitätsbibliotheken besucht werden, grundsätzlich ist aber eine bibliothekarische Tätigkeit nicht Voraussetzung für die Aufnahme in den Universitätslehrgang.

Zur Sicherstellung der wissenschaftlichen Qualität dieser Ausbildung gibt es an jeder der durchführenden Universitäten einen wissenschaftlichen Leiter (an der Uni Graz ist dies ein Mitarbeiter des ehemaligen Instituts für Informationswissenschaft). Österreichweit wurde ein sogenanntes wissenschaftliches Leitungsgremium installiert, das sich aus den wissenschaftlichen Leitenden der einzelnen Universitäten zusammensetzt und das einmal jährlich tagt.

\section{Literatur}

AM (2016). Grove Andrew, Sonnenwald Diane H., Harrison Lauren, Blake Catherine, Schlögl Christian, Peters Isabella, Endler-Jobst Barbara (Hg.) (2016). Creating Knowledge, Enhancing Lives through Information \& Technology. Proceedings of the 79th ASIS\&T Annual Meeting - Copenhagen, Denmark October, 2016. https://dl.acm.org/doi/proceedings/10.5555/3017447 [12.10.2020].

Bauer Gunter, Milchrahm Elisabeth, Rauch Wolf, Reichmann Gerhard \& Schlögl Christian (2005). Informationswissenschaft an der Karl-Franzens-Universität Graz, in: Eibl M., Wolff C., WomserHacker C. (Hg.): Designing Information Systems, Konstanz. Univ.-Verl. Konstanz, 1-12.

Dalpiaz, F. (2020). Research Challenges in Information Science, in: Proceedings of the 14th International Conference, RCIS 2020, Vol. 385. Springer Nature.

Dora, M. \& Kumar, H. A. (2019). National and international trends in library and information science research: A comparative review of the literature, in: IFLA Journal, DOI: https://doi.org/10.1177/ 0340035219886610.

Dorsch Isabella, Schlögl Christian, Stock Wolfgang G. \& Rauch Wolf (2017). Forschungsthemen der Düsseldorfer und Grazer Informationswissenschaft (2010 bis 2016), in: Information - Wissenschaft und Praxis 68(5-6), 320-328.

GLOMIS (o.D.). Global Studies on Management and Information Science (GLOMIS). https://studien.uni-graz.at/de/ordentlichestudien/global-studies-on-management-and-information-sci ence-glomis/global-studies-on-management-and-informationscience-glomis/ [5.9.2020].

HFDT (o.D.). HFDT Human Factor in Digtal Transition. https://digitaltransformation.uni-graz.at/de/ueber-das-netzwerk/ [5.9.2020].

Hörzer Birgit, Lach Karin, Pum Gabriele, Rabl-Altrichter Sylvia, Rezniczek Alina, Schlögl Christian, Schneider-Jakob Monika \& Seissl Maria (2020). Education for academic librarians: From the early beginnings up to the present, in: Education for Information, Preprint, 15 Seiten, DOI: 10.3233/EFI-190343.

Hsiao, T. M. \& Chen, K. H. (2020). The dynamics of research subfields for library and information science: an investigation based on word bibliographic coupling, in: Scientometrics, 125(1), 717-737.

ISI (1994). Dblp: computer science bibliography. https://dblp.org/ $\mathrm{db} /$ conf/isdiwi/isiwi1994.html [5.9.2020].

ISI (2015). 14th International Symposium on Information Science (ISI 2015). http://isi2015.de [5.9.2020].

Kolp, M., Snoeck, M., Vanderdonckt, J. \& Wautelet, Y. (2019, May). An Overview of Scientific Areas in Research Challenges in Information Science, in: Proceedings of the 13th International Conference on Research Challenges in Information Science (RCIS), 1-5.

Larivière, V., Sugimoto, C. R. \& Cronin, B. (2012). A bibliometric chronicling of library and information science's first hundred years, in: Journal of the American Society for Information Science and Technology, 63(5), 997-1016.

Luidold Kerstin, Seo DongBack \& Schlögl Christian (2016). Customers' Complaint Behavior on Social Media: Individualism versus Collectivism, in: Proceedings of the 16th International Conference on IT Applications and Management, $5 \mathrm{p}$.

Marchionini, G. (2017). Information science roles in the emerging field of data science, in: Journal of Data and Information Science 1(2), 1-6.

Maurer Angela, Schlögl Christian \& Dreisiebner Stefan (2016). Comparing information literacy of student beginners among different branches of study, in: Libellarium 9(2), 309-319.

Ortiz-Repiso, V., Greenberg, J. \& Calzada-Prado, J. (2018). A crossinstitutional analysis of data-related curricula in information science programmes: A focused look at the iSchools, in: Journal of Information Science, 44(6), 768-784.

Petrovic Otto \& Platzer Elisabeth (2009). Verbreitung von Mobilfunkdiensten und Erklärungsansätze für ihre Akzeptanz, in: Information - Wissenschaft und Praxis 60(1), 35-44.

Petrovic Otto, Puchleitner Thomas \& Harnisch Michael (2014). Research on Mobile Communication Systems in Last-Mile Logistics to Create and End-to- End Information Flow, in: International Journal of Services and Operations Management 19(2), 172-160.

Platzer Elisabeth, Petrovic Otto \& Rauch Wolf (2010). Causes and consequences of mobile phone's indispensability for everyday life, in: Proceedings of the 2010 Ninth International Conference on Mobile Business, 251-257.

Provost, F. \& Fawcett, T. (2013). Data science and its relationship to big data and data-driven decision making, in: Big data, 1(1), 51-59.

Rauch Wolf (2000). Bibliothek - Wozu?, in: B.I.T.-online - Zeitschrift für Bibliothek, Information und Technologie 3(4), 401-408.

Rauch Wolf (2016). Technological Change in Information and Communication, in: Bednar-Friedl Birgit \& Kleinert Jörn (Hg.). Dynamic Approaches to Global Economic Challenges - Festschrift in Honor of Karl Farmer. Heidelberg et al.: Springer, 131-140.

Reichmann Gerhard (2004). Measuring University Library Efficiency Using Data Envelopment Analysis, in: Libri - International Journal of Libraries and Information Services 54(2), 136-146.

Reichmann Gerhard (2007). Evaluation informationswissenschaftlicher Lehrveranstaltungen: Eine Längsschnittuntersuchung, in: Information - Wissenschaft und Praxis (nfd) 59(1), 21-27.

Reichmann Gerhard (2008). Welche Kompetenzen sollten gute Universitätslehrer aus der Sicht von Studierenden aufweisen? Ergebnisse einer Conjointanalyse, in: Das Hochschulwesen (HSW) 56(2), 52-57. 
Reichmann Gerhard (2012). Informationswissenschaft als Spezialisierung im Rahmen von betriebswirtschaftlichen Studiengängen an der Universität Graz - Eine empirische Studie, in: Petrovic, O./Reichmann, G./Schlögl C. (Hg.): Informationswissenschaft - Begegnungen mit Wolf Rauch, Wien: Böhlau, 359-374.

Reichmann Gerhard (2013). Textplagiate in der Wissenschaft und deren Verhinderung, in: Information - Wissenschaft und Praxis (nfd) 64(4), 175-184.

Reichmann Gerhard \& Sommersguter-Reichmann Margit (2012). The Austrian Tobacco Act in practice - Analysing the effectiveness of partial smoking bans in Austrian restaurants and bars, in: Health Policy 104(3), 304-311.

Schlögl Christian (2003). Wissenschaftslandkarte Informationsmanagement, in: Wirtschaftsinformatik 45(1), 7-16.

Schlögl Christian (2004). Die Wirtschaftsinformatik aus der Sicht ihres Hauptpublikationsorgans: Eine szientometrische Analyse der Zeitschrift Wirtschaftsinformatik/Angewandte Informatik, in: Wirtschaftsinformatik 46(4), 302-310.

Schlögl Christian (2013). Internationale Sichtbarkeit der europäischen und insbesondere der deutschsprachigen Informationswissenschaft, in: Information - Wissenschaft und Praxis 64(1), 1-8.

Schlögl Christian (2014). Comparison of downloads, citations and readership data for two information systems journals, in: Scientometrics 101(2), 1113-1128.

Uni Graz (o.D.). Die Universität Graz - Forschungsprofil. https:// www.uni-graz.at/de/die-universitaet/die-universitaet-graz/for schungsprofil/ [5.9.2020].

Virkus, S. \& Garoufallou, E. (2019). Data science from a library and information science perspective, in: Data Technologies and Applications 53(4), 422-441.

Wang, L. (2018). Twinning data science with information science in schools of library and information science, in: Journal of Documentation, 74(6), 1243-1257.

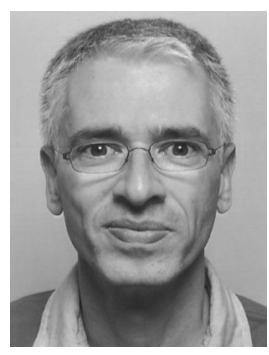

\section{Ao. Prof. DDr. Gerhard Reichmann}

Karl-Franzens-Universität Graz

Institut für Operations und Information

Systems

Universitätsstraße 15, F3

8010 Graz

gerhard.reichmann@uni-graz.at

Ao. Prof. DDr. Gerhard Reichmann ist seit 1997 an der Sozial- und Wirtschaftswissenschaftlichen Fakultät der Karl-Franzens-Universität Graz beschäftigt. Seine aktuellen Forschungsschwerpunkte sind Evaluierung (Evaluierung von universitärer Forschung, Lehre und Verwaltung, Evaluierung im Gesundheitsbereich), Informationskompetenz und Informationsrecht (Datenschutzrecht, Urheberrecht und Plagiatsproblematik).

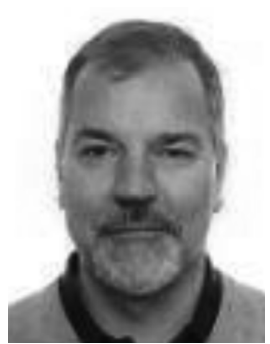

\author{
Ao. Prof. Dr. Christian Schlögl
}

Karl-Franzens-Universität Graz

Institut für Operations und Information

Systems

Universitätsstraße 15/F3

8010 Graz

christian.schloegl@uni-graz.at

Ao. Prof. Dr. Christian Schlögl ist seit 1990 an der Sozial- und Wirtschaftswissenschaftlichen Fakultät der Karl-Franzens-Universität Graz beschäftigt, seit 2005 ist er stellvertretender wissenschaftlicher Leiter des an den Universitäten Wien, Innsbruck und Graz eingerichteten interuniversitären Universitätslehrgangs „Master of Science (MSc) in Library and Information Studies“. Seine aktuellen Forschungsgebiete sind Bibliometrie und Szientometrie, Informationskompetenz sowie Informations- und Wissensmanagement.

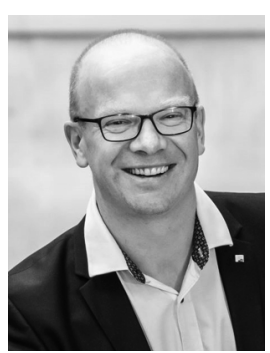

Prof. Dr. Stefan Thalmann

Karl-Franzens-Universität Graz

Institut für Operations und Information

Systems

Universitätsstraße 15/F3

8010 Graz

stefan.thalmann@uni-graz.at

Univ.-Prof. Dr. Stefan Thalmann seit 2018 an der Universität Graz, Leiter des Business Analytics und Data Science Centers und Bereichsleiter am Institut für Operations und Information Systems. Nach dem Studium der Wirtschaftsinformatik an der Martin-Luther Universität Halle-Wittenberg hat er an der Universität Innsbruck im Bereich Wirtschaftsinformatik promoviert und habilitiert. AnschlieBend war er an der TU Graz und hat dort im Forschungscenter Pro2Future eine Arbeitsgruppe zu Industrial Data Analytics geleitet. Seite Forschungsgebiete sind Business Analytics, Datenbasierte Kollaborationen, Wissensrisiken und XAI. 